GrigG, G. W. (1960). J. gen. Microbiol. 22, 667-670

\title{
Temperature-sensitive Genes Affecting Conidiation in Neurospora
}

\author{
By G. W. GRIGG \\ Animal Genetics Section (C.S.I.R.O.), Zoology Department, University of \\ Sydney, Australia
}

SUMMARY: Two temperature-sensitive genes are described which affect conidiation in Neurospora crassa. One (acont) decreases the length of aerial hyphae at

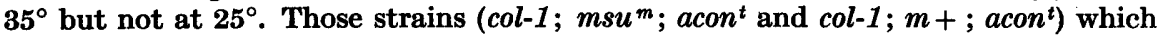
have short aerial hyphae at $2^{\circ}$ are aconidiate at $35^{\circ}$.

Growth on a rich conidiating medium results in the non-expression of $a^{c o n}{ }^{t}$ in some but not all of the $a c{ }^{t}$ strains tested. To explain the observations on conidiation in the $a \operatorname{con}^{t}$ strains it is suggested that $a{ }^{2}{ }^{t}$ is active during a certain period in the ontogeny of the culture, this period being independent of genotype and environment.

Another genotype col-1; $m$; acon ${ }^{t+}$ has a phenotype which is dependent on the incubation temperature. At $25^{\circ}$ it is microconidiate but at $35^{\circ}$ it is macroconidiate. Since col-1; $m^{+}$strains are aconidiate, the $m$ gene determines whether any conidia shall be produced, but the type of conidia formed is determined by the ambient temperature. The $s u^{m}$ gene removes this environmental control of conidial phenotype, the col-1;m su $u^{m}$ strains being macroconidiate at both temperatures. The $s u^{m}$ gene may be described therefore as a canalization gene.

The conidiation of Neurospora cultures is affected both quantitatively and qualitatively by the genotype and the environment (e.g. Barrett \& Garnjobst, 1949; Grigg, 1958, 1960). It is the purpose of this paper to describe the effect of temperature on two genes affecting conidiation in Neurospora crassa. One gene, the well-known peach-microconidial ( $p e^{m}$ of Barratt \& Garnjobst, 1949, referred to hereafter as $m$ ) will be mentioned only briefly; the other, previously undescribed, will be dealt with in more detail. The $m$ gene in a wild-type background affects the colour and the morphology of the culture. Barratt \& Garnjobst described it as 'peach clumpy'. The long aerial hyphae on which the macroconidia form in wild type cultures are produced at the open end of tube slopes. In clumpy strains the lengths of the aerial hyphae are shorter than in wild type and are distributed over the entire surface of the culture. Consequently, unlike the wild type cultures, the $m$ (clumpy) one produces macroconidia over the entire culture surface. The strains and methods have been described in a previous paper (Grigg, 1960).

\section{RESULTS}

\section{Peach microconidial gene $(m)$}

col-1; $m^{+}$cultures are ahyphal and virtually aconidiate at $25^{\circ}$ (and $35^{\circ}$ ), but col-1; $m$ strains are microconidiate at $25^{\circ}$ (Barratt \& Garnjobst, 1949). At $3^{\circ}$ however, col-1; $m$ cultures were observed to be macroconidiate and resembled the phenotype of $c o l-1 ; m s u^{m}$ cultures. Thus although $m$ must be 
present in a col-1 strain for conidia to be produced at all, the type of conidia produced seems to be controlled by the temperature at which the culture is grown.

\section{Temperature-sensitive aconidiate gene (acon ${ }^{t}$ )}

The observation that one of the laboratory cultures had an appearance and conidiation which was different at $35^{\circ}$ from that at $25^{\circ}$ led to a survey of the appearances of other stock cultures at the two temperatures. The stocks could be grouped into those which were unaffected by the higher temperature and those which produced shorter aerial hyphae at the higher temperature. In this latter group conidiation was depressed, the extent of the inhibition depending on the background genotype (Table 1 ). Those macroconidial strains, such as some of the col-1; $m s u^{m}$ stocks, which had short hyphae at $25^{\circ}$ were virtually aconidiate at $35^{\circ}$. This effect of temperature on length of aerial hyphae segregated regularly as a single gene in ordered asci and random spore analysis. No linkage was detected with $m$ or col-1.

Table 1. Morphology of temperature sensitive (acont) strains at $25^{\circ}$ and $35^{\circ}$

\begin{tabular}{|c|c|c|}
\hline \multirow[b]{2}{*}{ Background genotype } & \multicolumn{2}{|l|}{ Morphology } \\
\hline & $25^{\circ}$ & $\mathbf{3 5}^{\circ}$ \\
\hline$+;++(+\mathbf{a})$ & $\begin{array}{l}\text { Wild-type. Macroconidia } \\
\text { produced on long aerial } \\
\text { hyphae at plug end of tube } \\
\text { slope }\end{array}$ & 'Clumpy' \\
\hline$+; m+(\mathbf{m a})$ & $\begin{array}{l}\text { 'Clumpy'. Macroconidia pro- } \\
\text { duced on shortened aerial } \\
\text { hyphae over entire culture } \\
\text { surface }\end{array}$ & 'cms' \\
\hline col-1;msu $u^{m}$ (cmsa) & $\begin{array}{l}\text { 'cms'. Macroconidia produced } \\
\text { on very short hyphae over } \\
\text { entire culture surface }\end{array}$ & Aconidiate \\
\hline $\operatorname{col}-1 ; m+(\mathbf{c m a})$ & $\begin{array}{l}\text { 'cm'. No aerial hyphae. Micro- } \\
\text { conidia produced over culture } \\
\text { surface }\end{array}$ & Aconidiate \\
\hline $\operatorname{col}-1 ;++(c a)$ & Aconidiate. No aerial hyphae & Aconidiate \\
\hline
\end{tabular}

At $35^{\circ}$ the temperature-sensitive $+;++(+a)$ cultures had the clumpy phenotype characteristic of $+; m+$ strains ( $m, \mathbf{m a})$ grown at $25^{\circ}$ and the temperature-sensitive $+; m+$ cultures (ma) had a colonial, velvety phenotype similar in appearance to that of col-1, $m \mathrm{su}^{m}$ strains (cms, cmsa) at $25^{\circ}$. The temperature-sensitive col-1; $m+(\mathrm{cma})$ and col-1 $m s u^{m}$ (cmsa) strains were aconidiate at $\mathbf{3 5}^{\circ}$.

Because of its extreme effect on condiation of cma and cmsa strains at $35^{\circ}$ the gene has been designated 'aconidiate temperature sensitive' $\left(a c o n{ }^{t}\right)$.

\section{Interaction of temperature and medium on conidiation of acont strains}

That the medium on which a Neurospora culture is grown affects macroconidiation is well known. In general, media with a high nitrogen content and glycerol as a carbohydrate source favour conidiation; an excellent example 
of this is Horowitz's (1947) conidiating medium. We found that microconidiation is enhanced on medium supplemented with casein hydrolysate (Grigg, 1960). In certain genetic backgrounds the medium on which the culture was grown influenced the expression of the $a^{a c o n}{ }^{t}$ gene. Thus the phenotypes of the $a^{a c o n}{ }^{t}$ strains, +a, ma and cmsa resembled those of the corresponding acont ${ }^{t+}$ ones when the former were grown on conidiating medium. In the col-1;m background genotype the expression of the acon ${ }^{t}$ gene was unaltered by growth in conidiating medium. The phenotype of the cma strain at $35^{\circ}$ was aconidiate on all media. At $25^{\circ}$ this strain was, of course, microconidiate on both minimal and complete media. The effect of the complete medium on the acon ${ }^{t}$ cannot therefore be ascribed to supplying a specific growth requirement of the $a^{a}{ }^{t}$ gene. Moreover, supplementing the medium with a wide range of common growth factors including those in glycerol $(2 \%)$, malt extract $2 \%$, casein hydrolysate $(1.4 \%)$, nucleic acid hydrolysate $(0.4 \%)$, yeast extract $(1 \cdot 4 \%)$ or a combination of all of these (excepting malt extract) did not affect the $a^{t}{ }^{t}$ character of any of the $a^{2}{ }^{t}$ strains.

\section{DISCUSSION}

Time of expression of acon ${ }^{t}$. The exact effect of $a^{2}{ }^{t}$ on conidial production at $35^{\circ}$ varied with the background genotype. Thus on minimal medium conidial production of the two colonial strains cma and cmsa were completely suppressed, that of ma (clumpy) somewhat restricted and that of $+a$ scarcely affected. The effect on the conidial character of growing these strains on conidiating medium followed the same progression. The cma strain remained aconidiate, but the acon ${ }^{t}$ character of the cmsa, ma and $+\mathbf{a}$ strains was not expressed. Now the period between inoculation and conidiation varies from strain to strain in the following way: cma $>$ cmsa $>$ ma $>+a$ (Fig. 1). Conidiating medium decreases this period for all but the cma strain. To explain these observations it is suggested that either acon ${ }^{t}$ is active only during a discrete period in development or the organism is capable of reacting to a stimulus provided by acon ${ }^{t}$ only during a particular short period. This period $\left(t_{1}\right.$, Fig. 1) must be independent of genotype and environment. The hypothesis is shown diagrammatically in Fig. 1.

The blocking effect of acon $^{t}$ is reversible since transfer of, for example, a cmsa or a cma culture from $35^{\circ}$ to $25^{\circ}$ results in conidial formation on the previously aconidiate cultures. The type of conidia produced by a culture seems to be determined during a definite period in development (Grigg, 1960), the exact position of the critical period being controlled by the genotype.

Canalization gene affecting conidiation. The environmental control of the conidial phenotype of the $\mathbf{c m}$ strain is complete. At $25^{\circ}$ the culture is microconidiate. If, however, the $s u^{m}$ gene is present (Grigg, 1958) the sensitivity of the $\mathbf{c m}$ phenotype to temperature is no longer apparent. The $s u^{m}$ strain $\mathbf{c m s}$ is macroconidiate at both $25^{\circ}$ and $35^{\circ}$. The gene $s u^{m}$ may be described therefore as a canalization gene (Waddington, 1957; and refs.), since no matter what the temperature, the conidial character is invariate. As far as we know this 
'recessive' gene affects no character other than the conidial one. It has some selective advantage since stock cultures which originally were col-1;msu+ changed to the col-1; $m s u^{m}$ genotype in the course of several subcultures.
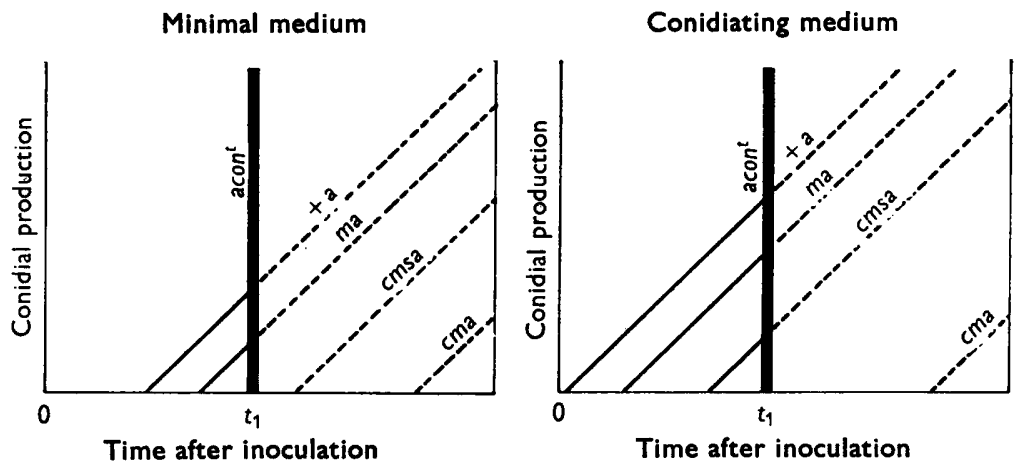

Fig. 1. Diagram to illustrate conidial production of strains +a, ma, cmsa and cma on minimal and conidiating medium. The gene $a_{c o n}{ }^{t}$ is represented as blocking conidial production at a time $t_{1}$, after inoculation at time 0 .

\section{REFERENCES}

Barratt, R. W. \& Garnjobst, L. (1949). Genetics of a colonial microconidiating mutant strain of Neurospora crassa. Genetics, 34, 351.

Grige, G. W. (1958). The genetic control of conidiation in a heterocaryon of Neurospora crassa. J. gen. Microbiol. 19, 15.

GrIGg, G. W. (1960). The control of conidial differentiation in Neurospora crassa. J. gen. Microbiol. 22, 663.

Horowitz, N. H. (1947). Methionine synthesis in Neurospora: The isolation of cystathionine. J. biol. Chem. 171, 255.

Waddington, C. H. (1957). The strategy of the gene. London: Allen and Unwin.

(Received 26 October 1959) 